\title{
Lipids from the rhizome of Cnidium officinalis Makino
}

\author{
Hyoung-Geun Kim ${ }^{1}$ (D) $\cdot$ Hyeong-Ju Jeon ${ }^{2}$ (D) $\cdot$ Trong Nguyen Nguyen ${ }^{1} \cdot$ \\ Dae Young Lee $^{3}$ (D) $\cdot$ Nam-In Baek ${ }^{1}$ (D)
}

\section{천궁으로부터 lipid 의 분리 동정}

\author{
김형근 ${ }^{1} \cdot$ 전형주 $^{2} \cdot$ 웅우옌 트롱 웅우옌 ${ }^{1} \cdot$ 이대영 ${ }^{3} \cdot$ 백남인 $^{1}$
}

Received: 26 August 2021 / Accepted: 24 September 2021 / Published Online: 31 December 2021

(C) The Korean Society for Applied Biological Chemistry 2021

\begin{abstract}
The rhizomes of Cnidium officinalis were extracted in aqueous $\mathrm{MeOH}$, and the concentrate was fractionated via systematic solvent fractionation to EtOAc, $n-\mathrm{BuOH}$, and aqueous fractions. The repeated column chromatography of EtOAc and $n$ $\mathrm{BuOH}$ fractions using silica gel, octadecyl silica gel, and Sephadex LH-20 as stationary phase to afford five lipids. They were identified to be methyl linoleate (1), linoleic aicd (2) 6linoleoyl- $\alpha$-D-glucopyranosyl $\beta$-D-fructofuranoside (3), 1-linolenoyl3-( $\alpha$-D-galactopyranosyl $(1 \rightarrow 6)-\beta$-D-galactopyranosyl) glycerol (4), and 1-linoleoyl-3-( $\alpha$-D-galactopyranosyl $(1 \rightarrow 6)-\beta$-D-galactopyranosyl) glycerol (5) on the basis of spectroscopic data such as IR, MS, and Nuclear magnetic resonance (NMR). Compounds 1 and 3-5 were isolated for the first time from this plant in this study. The NMR data of fatty acids $\mathbf{1}$ and $\mathbf{2}$ reported in literatures are different each other. Authors identified the NMR data without ambiguity. Compound 3, a conjugate of sucrose and fatty acid, and compounds $\mathbf{4}$ and $\mathbf{5}$, digalactosyl monoglyceride, are very rarely occurred in natural source. Through the immune
\end{abstract}

Nam-In Baek $(\bowtie)$

E-mail:nibaek@khu.ac.kr

${ }^{1}$ Graduate School of Biotechnology and Department of Oriental Medicinal Biotechnology, Kyung Hee University, Yongin 17104, Republic of Korea

${ }^{2}$ Department of Food \& Nutrition, Jangan University, Hwaseong 18331, Republic of Korea

${ }^{3}$ Department of Herbal Crop Research, National Institute of Horticultural and Herbal Science, RDA, Eumseong 27709, Republic of Korea

This is an Open Access article distributed under the terms of the Creative Commons Attribution Non-Commercial License (http://creativecommons. org/licenses/by-nc/3.0/) which permits unrestricted non-commercial use, distribution, and reproduction in any medium, provided the original work is properly cited. enhancement and anticancer activity of the reported lipid compounds, the potential as various pharmacologically active materials of Cnidium officinalis rhizome can be expected.

Keywords Cnidium officinalis - Diglycosyl monoglyceride · Linoleoyl sucrose $\cdot$ Lipid $\cdot$ Nuclear magnetic resonance

서 론

천궁(Cnidium offininalis) 은 미나리과(Apiaceae)에 속하는 다년 생 초본식물로, $30-60 \mathrm{~cm}$ 로 자라며, 계란형 모양의 깃털을 가진 겹잎이 어긋난 형태로 되어 있다. 꽃은 작고 흰색이며, 타원형 의 꽃잎, 꽃받침, 수술이 모두 5개씩이다[1]. 중국 남부가 원산 이나 현재는 중국, 한국, 일본에서 널리 재배하고 있다. 천궁의 어린 잎은 나물로 먹으며[1], 근경은 혈관을 확장하여 혈액순환 을 활발하게 하고, 기운이 순조롭게 통하도록 한다 하여, 예로 부터 이와 관련된 다양한 질환의 예방과 치료에 널리 사용되어 왔다[2,3]. 최근의 연구에서도 천궁 추출물 및 그 함유성분이 항 암[4], 진통[5], 항박테리아[6], 항경련[7], 항염[4,5], 발열, 저혈 압[8], 진정[9], 그리고 혈관확장[10] 등의 약리활성이 있는 것 으로 보고되었다. 천궁의 근경으로부터 페놀산, 유기산, 지방산, phthalide, alkaloid 등의 성분이 분리 보고되고 있으며[1], 그 중 phthalide는 천궁의 가장 주요한 성분으로, 빈혈 개선, 진정[11], 항균[12], 항보체[13], 평활근 이완[14]과 같은 약리활성이 phthalide에서 기인하는 것으로 밝혀졌다. 본 실험에서는 천궁의 근경으로부터 5 종의 지질 화합물을 분리하고 동정하였고, 그 중 2 분자의 galactose가 결합한 glycosylglyceride 2종과 sucrose에 지방산이 결합한 화합물 1종은 천연에서 매우 드물게 보고되는 당지질이다. 본 논문에서는 5 종 지질 화합물의 분리, 정제 과정 과, Nuclear magnetic resonance (NMR), IR, MS와 같은 스펙 
트럼 데이터를 해석하여 구조를 결정하는 과정을 기술하였다.

\section{재료 및 방법}

\section{시험 약재}

본 실험에 사용한 천궁의 근경은 농촌진흥청 국립원예특작과학 원 약용작물과로부터 제공 받았으며, 정진태 연구사가 동정하였 다. 표준 시료는 경희대학교 천연물화학실험실에 보관되어 있다.

\section{시약 및 기기}

칼럼크로마토그래피에 사용한 충진제는 silica gel (Kiesel gel 60, Merck, Darmstadt, Germany), ocatdecyl silica gel (LiChroprep RP-18, 40-60 $\mu \mathrm{m}$, Merck)과 Sephadex LH-20 (Amersham Biosciences, Uppsala, Sweden)을 사용하였다. 박층크로마토그래 피는 Kiesel gel $60 \mathrm{~F}_{254}$ 와 $\mathrm{RP}-18 \mathrm{~F}_{254} \mathrm{~S}$ (Merck)를 사용하였으 며, UV lamp (Spectroline Model ENF-240 C/F, Spectronics Corporation, Westbury, NY, USA)를 사용하여 검출하였으며, $10 \%$ 황산을 적신 후 가열하여 발색하였다. ${ }^{1} \mathrm{H} \mathrm{NMR}$ 과 ${ }^{13} \mathrm{C}$ $\mathrm{NMR}$ 스펙트럼은 Bruker Avance 600 (Billerica, MA, USA) 로, IR 스펙트럼은 Perkin Elmer Spectrum One FT-IR spectrometer (Buckinghamshire, England)로, 질량분석 스펙트럼 은 JEOL JMSAX-700 mass spectrometer (Tokyo, Japan)로 측정하였다.

EtOAc fraction from the rhizomes of Cnidium officinalis (125 g)

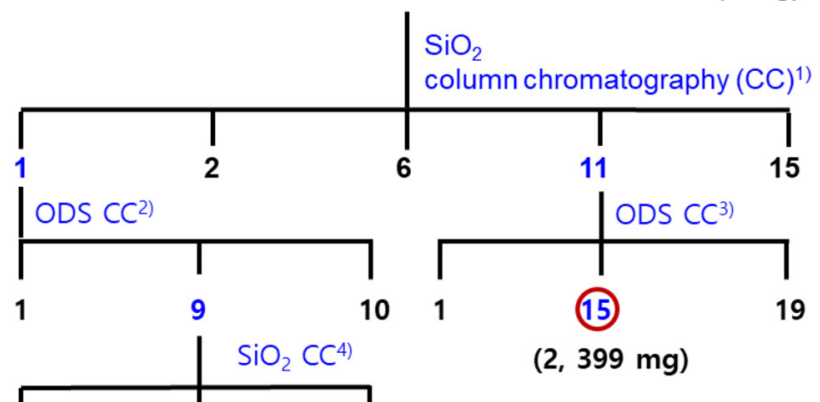

\footnotetext{
149

Sephadex LH-20 $\mathrm{CC}^{5}$ $(1,250 \mathrm{mg})$

6) Ø $11 \times 17 \mathrm{~cm} / \mathrm{CHCl}_{3}-\mathrm{MeOH}-\mathrm{H}_{2} \mathrm{O}=15: 3: 1 \rightarrow 10: 3: 1 \rightarrow 7: 3: 1$

7) $\varnothing 4.5 \times 7.0 \mathrm{~cm} / \mathrm{MeOH}-\mathrm{H}_{2} \mathrm{O}=3: 2$

8) $\varnothing 4.5 \times 7.0 \mathrm{~cm} / \mathrm{MeOH}-\mathrm{H}_{2} \mathrm{O}=1: 3 \rightarrow 1: 2 \rightarrow 1: 1$
}

\section{지질화합물의 분리}

건조한 천궁 근경 분말 $(10 \mathrm{~kg})$ 을 $70 \%$ ethanol $(\mathrm{EtOH}, 54 \mathrm{~L})$ 로 실온에서 15 시간 2 회 반복 추출하였다. 추출액을 여과하고, 여 액을 감압 농축하여 추출물 $(2.1 \mathrm{~kg})$ 을 얻었다. 추출물에 $\mathrm{H}_{2} \mathrm{O}$ $(4 \mathrm{~L})$ 을 넣어 현탁한 후, EtOAc $(4 \mathrm{~L} \times 3)$ 와 $n-\mathrm{BuOH}(3.2 \mathrm{~L} \times$ $3)$ 로 분획하였다. 얻어진 분획을 감압농축하여 EtOAc 분획물 $(\mathrm{COE}, 280 \mathrm{~g}), n-\mathrm{BuOH}$ 분획물 $(\mathrm{COB}, 125 \mathrm{~g})$ 및 물 분획물 $(\mathrm{COW}, 1.695 \mathrm{~kg})$ 을 제조하였다. EtOAc 분획물 $(270 \mathrm{~g})$ 과 $n-$ $\mathrm{BuOH}$ 분획물 $(120 \mathrm{~g})$ 에 대하여 silica gel, octadecyl silica gel 및 Sephadex LH-20을 정지상으로 사용하여 칼럼크로마토그래 피를 반복 수행한 결과 5 종의 지질화합물을 분리하였다(Fig. 1).

\section{Methyl linoleate (1)}

Colorless oil; TLC (silica gel) $R f$ 0.62, $n$-hexane-EtOAc (8:1), (octadecyl silica gel) $R f$ 0.38, acetone- $\mathrm{H}_{2} \mathrm{O} \quad(7: 1)$; IR (LiF plates) $v_{\max } 2924,2854,1749,1458,1436 \mathrm{~cm}^{-1} ;{ }^{13} \mathrm{C}$ 과 ${ }^{1} \mathrm{H}$ NMR 스펙트럼: Table 1과 2. EI-MS: $m / z 294[\mathrm{M}]^{+}$.

\section{Linoleic acid (2)}

Colorless oil; TLC (silica gel) Rf $0.69, n$-hexane-EtOAc (1:1), (octadecyl silica gel) $R f 0.83$, acetone- $\mathrm{H}_{2} \mathrm{O}$ (3:1); IR (LiF plates) $v_{\max } 2930,1740,1462,1438 \mathrm{~cm}^{-1} ;{ }^{13} \mathrm{C}$ 과 ${ }^{1} \mathrm{H}$ $\mathrm{NMR}$ 스펙트럼: Table 1과 2. EI-MS: $m / z 280[\mathrm{M}]^{+}$.
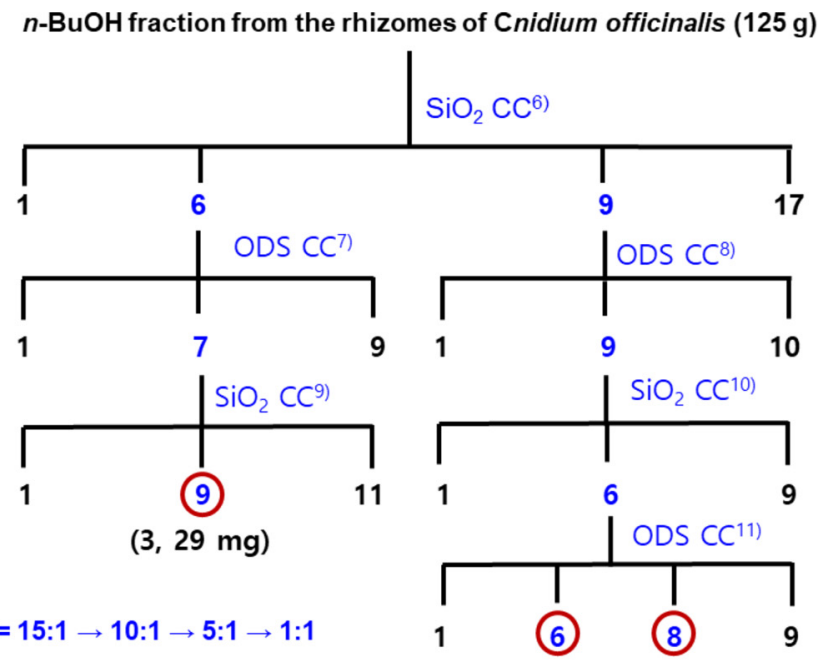

$(4,8 \mathrm{mg})(5,50 \mathrm{mg})$

\footnotetext{
1) Ø11 x $16 \mathrm{~cm} / \mathrm{n}$-hexane-EtOAc $=15: 1 \rightarrow 10: 1 \rightarrow 5: 1 \rightarrow 1: 1$

2) $\varnothing 4.5 \times 7.0 \mathrm{~cm} /$ acetone $-\mathrm{H}_{2} \mathrm{O}=3: 2$

3) $\varnothing 4.5 \times 7.0 \mathrm{~cm} /$ acetone $-\mathrm{H}_{2} \mathrm{O}=1: 2 \rightarrow 1: 1 \rightarrow 2: 1$

4) $\varnothing 3.0 \times 20 \mathrm{~cm} / n$-hexane-EtOAc $=30: 1$

5) $\varnothing 1.5 \times 55 \mathrm{~cm} / \mathrm{MeOH}$
}

9) $\varnothing 4.5 \times 7.0 \mathrm{~cm} / \mathrm{CHCl}_{3}-\mathrm{MeOH}-\mathrm{H}_{2} \mathrm{O}=10: 3: 1$

10) $\varnothing 5.0 \times 17 \mathrm{~cm} / \mathrm{CHCl}_{3}-\mathrm{MeOH}-\mathrm{H}_{2} \mathrm{O}=10: 3: 1 \rightarrow 8: 3: 1 \rightarrow 65: 35: 10$

11) $\varnothing 3.0 \times 9.0 \mathrm{~cm} /$ acetone- $\mathrm{H}_{2} \mathrm{O}=3: 2$

Fig. 1 Isolation procedure of lipids from the rhizome of Cnidium officinalis 


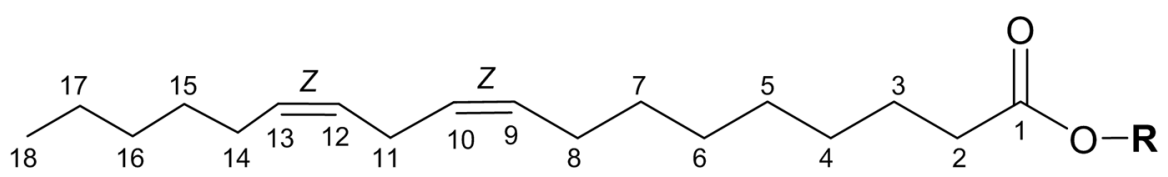

\section{1: $\mathrm{R}=\mathrm{H} \quad$ 2: $\mathrm{R}=\mathrm{CH}_{3}$}

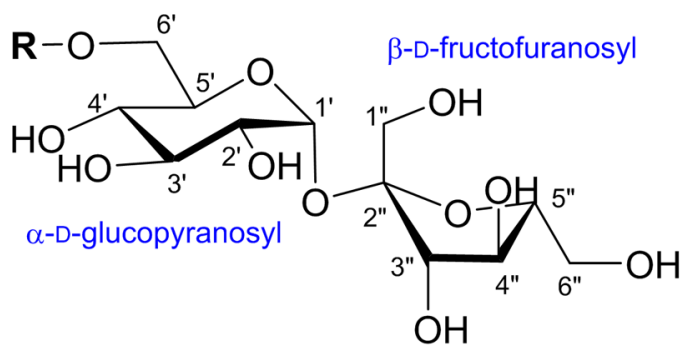

3: $\mathrm{R}=$ linoleic acid

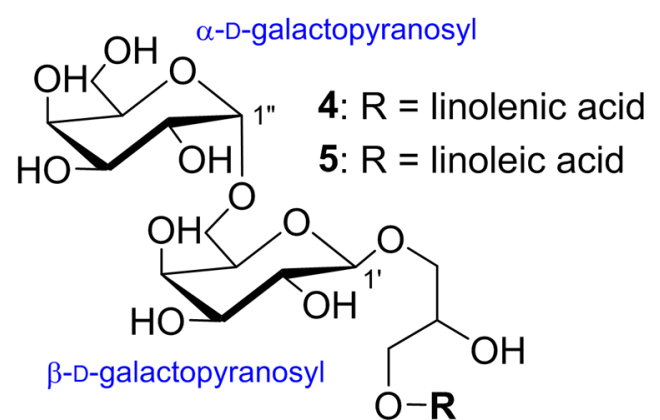

Fig. 2 Chemical structure of lipids from the rhizome of Cnidium officinalis. 1, methyl linoleate; 2, linoleic aicd; 3, 6-linoleoyl- $\alpha$-D-Glucopyranosyl $\beta$ D-fructofuranoside; 4, 1-linolenoyl-3-( $\alpha$-D-galactopyranosyl $(1 \rightarrow 6)-\beta$-D-galactopyranosyl) glycerol; 5, 1 -linoleoyl-3-( $\alpha$-D-galactopyranosyl $(1 \rightarrow 6)-\beta$ D-galactopyranosyl) glycerol

6-Linoleoyl- $\boldsymbol{\alpha}$-D-glucopyranosyl $\boldsymbol{\beta}$-D-fructofuranoside (3) White powder; TLC (silica gel) $R f$ 0.44, $\mathrm{CHCl}_{3}-\mathrm{MeOH}-\mathrm{H}_{2} \mathrm{O}$ (7:3:1), (octadecyl silica gel) $R f 0.45, \mathrm{MeOH}-\mathrm{H}_{2} \mathrm{O}$ (5:1); IR (LiF plates) $v_{\max } 3331,2924,2854,1721,1457,1378 \mathrm{~cm}^{-1}$; ${ }^{13} \mathrm{C}$ 과 ${ }^{1} \mathrm{H}$ NMR 스펙트럼: Table 1 과 2. positive FAB-MS: $m / \mathrm{z} 605[\mathrm{M}+\mathrm{H}]^{+}$.

\section{1-Linolenoyl-3-( $\alpha$-D-galactopyranosyl $(1 \rightarrow 6)-\beta$-D-galactopyranosyl) glycerol (4)}

White powder; TLC (silica gel) $R f 0.43, \mathrm{CHCl}_{3}-\mathrm{MeOH}-\mathrm{H}_{2} \mathrm{O}$ (65:35:10), (octadecyl silica gel) $R f$ 0.62, acetone- $\mathrm{H}_{2} \mathrm{O}(5: 1)$; IR (LiF plates) $v_{\max } 3343,2921,1732,1456,1376 \mathrm{~cm}^{-1} ;{ }^{13} \mathrm{C}$ 과 ${ }^{1} \mathrm{H}$ NMR 스펙트럼: Table 1과 2. positive FAB-MS: $m / z$ $677[\mathrm{M}+\mathrm{H}]^{+}$.

\section{1-Linoleoyl-3-( $\alpha$-D-galactopyranosyl $\quad(1 \rightarrow 6)-\beta$-D-galactopyranosyl) glycerol (5)}

White powder; TLC (silica gel) $R f$ 0.43, $\mathrm{CHCl}_{3}-\mathrm{MeOH}-\mathrm{H}_{2} \mathrm{O}$ (65:35:10), (octadecyl silica gel) $R f 0.59$, acetone- $\mathrm{H}_{2} \mathrm{O}(3: 1)$; IR (LiF plates) $v_{\max } 3347,2923,1732,1648,1377 \mathrm{~cm}^{-1} ;{ }^{13} \mathrm{C}$ 과 ${ }^{1} \mathrm{H}$ NMR 스펙트럼: Table 1 과 2. positive FAB-MS: $m / z$ $679[\mathrm{M}+\mathrm{H}]^{+}$.

\section{결과 및 고찰}

천궁의 근경을 $\mathrm{EtOH}$ 로 추출하였으며, 얻어진 추출물을 용매 의 극성을 이용하여 EtOAc, $n-\mathrm{BuOH}$ 및 물 분획으로 제조하였 다. 그 중 $\mathrm{EtOAc}$ 와 $n-\mathrm{BuOH}$ 분획에 대하여 silical gel, octadecyl silica gel 및 Sephadex LH-20을 정지상으로 사용하
여 칼럼크로마토그래피를 반복 수행한 결과 5종의 지질화합물 1-5를 분리하였다.

화합물 1과 2는 ${ }^{13} \mathrm{C} \mathrm{NMR}$ 스펙트럼에서 methyl ester의 methyl기를 제외하면 모두 18 개의 탄소 signal이 관측되었다. 한 개의 carboxyl 기 혹은 ester 탄소, 4개의 olefin methine 탄소, 12 개의 methylene 탄소, 1 개의 methyl 탄소에서 유래한 signal 이 관측됨에 따라 이 화합물들은 octadecadienoic acid 로 추정 되었다. NMR, MS, IR 데이터를 문헌 값과 비교하여 화합물 1 과 2를 각각 methyl linoleate (methyl 9Z,12Z-octadeca-9,12dienoeate)와 linoleic acid (9Z,12Z-octadeca-9,12-dienoic acid) 로 구조 동정하였다. 지금까지 문헌에 보고된 각 화합물의 $\mathrm{NMR}$ 데이터를 보면, 일부의 탄소와 수소에 대해서는 빠져 있 던가[15], 혹은 문헌 마다 서로 다른 값으로 보고되어 있다. 저 자 등은 이번에 ${ }^{1} \mathrm{H}-{ }^{1} \mathrm{H}$ COSY, HSQC 및 $\mathrm{HMBC}$ 와 같은 $2 \mathrm{D}-$ $\mathrm{NMR}$ 스펙트럼을 자세히 검토하여 모든 탄소와 수소에 대한 데 이터를 정확하게 동정하였다.

화합물 3은 positive FAB-MS 스펙트럼에서 분자이온피크가 $m / z 605$ 에서 관측되어 분자량을 $604 \mathrm{Da}$ 로 결정하였다. IR 스펙 트럼에서 수산기 $\left(3331 \mathrm{~cm}^{-1}\right)$, ester $\left(1721 \mathrm{~cm}^{-1}\right)$ 및 olefin (1457, $\left.1378 \mathrm{~cm}^{-1}\right)$ 에서 유래한 흡수밴드가 확인되었다. NMR 스펙트럼 을 보면 모두 30 개의 탄소 signal이 관측되었고, 각 signal의 chemical shift 로부터 2분자의 hexose와 18 개의 탄소로 이루어 진 1 분자의 지방산이 결합한 당지질 화합물로 확인되었다. 당 에서 유래한 signal 을 보면 1개의 hemiacetal $\left(\delta_{\mathrm{C}}\right.$ 93.71, C-1'; $\delta_{\mathrm{H}} 6.21$, br.s, $\left.\mathrm{H}-1^{\prime}\right)$, 1 개의 hemiketal $\left(\delta_{\mathrm{C}} 106.35, \mathrm{C}-2^{\prime \prime}\right)$, 7개 의 oxygenated-methine $\left(\delta_{\mathrm{C}} 73.89, \mathrm{C}-2^{\prime} ; 75.52, \mathrm{C}-3^{\prime} ; 72.46\right.$, C-4'; 75.08, C-5'; 79.79, C-3"; 77.09, C-4"; 81.25, C-5"; $\delta_{\mathrm{H}}$ 4.20-5.01, H-2' 5', H-3" ( $\left.^{\prime \prime}\right)$, 3개의 oxygenated-methylene $\left(\delta_{\mathrm{C}}\right.$ 67.08, С-6'; 63.09, C-1"; 64.82, C-6"; $\delta_{\mathrm{H}}$ 4.36-5.06, H-6', $\mathrm{H}-1$ ", $\left.\mathrm{H}-6^{\prime \prime}\right)$ 이 관측된 점으로부터 aldohexose와 ketohexose가 
Table $1{ }^{13} \mathrm{C}$ NMR data of lipids from the rhizome of Cnidium officinale Makino $\left(125 \mathrm{MHz}, \delta_{\mathrm{C}}\right)$

\begin{tabular}{|c|c|c|c|c|c|}
\hline \multirow{2}{*}{ No of C } & \multicolumn{5}{|c|}{ Lipids* } \\
\hline & $\mathbf{1}\left(\mathrm{CDCl}_{3}\right)$ & $2\left(\mathrm{CDCl}_{3}\right)$ & $\boldsymbol{3}\left(\mathrm{CD}_{3} \mathrm{OD}\right)$ & $4\left(\mathrm{CD}_{3} \mathrm{OD}\right)$ & $\mathbf{5}\left(\mathrm{CD}_{3} \mathrm{OD}\right)$ \\
\hline 1 & 174.40 & 180.64 & 173.98 & 175.63 & 175.61 \\
\hline 2 & 34.27 & 34.31 & 34.63 & 35.07 & 35.07 \\
\hline 3 & 25.15 & 24.85 & 25.51 & 26.13 & 26.13 \\
\hline 4 & 29.30 & 29.26 & 29.72 & 30.34 & 30.37 \\
\hline 5 & 29.27 & 29.22 & 29.18 & 30.36 & 30.34 \\
\hline 6 & 29.33 & 29.33 & 29.83 & 30.85 & 30.46 \\
\hline 7 & 29.83 & 29.77 & 30.24 & 30.46 & 30.86 \\
\hline 8 & $27.39^{\#}$ & $27.39^{\#}$ & $27.85^{\#}$ & 28.31 & 28.30 \\
\hline 9 & 130.19 & 130.16 & 130.78 & 132.88 & 131.02 \\
\hline 10 & 128.24 & 128.26 & $128.75^{\# \#}$ & 128.40 & 129.25 \\
\hline 11 & 25.82 & 25.82 & 26.38 & 26.55 & 26.68 \\
\hline 12 & 128.11 & 128.09 & $128.75^{\# \#}$ & 129.36 & 129.19 \\
\hline 13 & 130.36 & 130.35 & 130.80 & 129.35 & 131.08 \\
\hline 14 & $27.37^{\#}$ & $27.36^{\#}$ & $27.82^{\#}$ & 26.67 & 28.30 \\
\hline 15 & 29.53 & 29.54 & 29.94 & 129.01 & 30.62 \\
\hline 16 & 31.71 & 31.72 & 32.03 & 131.24 & 32.81 \\
\hline 17 & 22.74 & 22.76 & 23.15 & 21.64 & 23.77 \\
\hline 18 & 14.20 & 14.22 & 14.57 & 14.81 & 14.59 \\
\hline $\mathrm{OMe}$ & 51.53 & - & - & - & \\
\hline Glycerol-1 & - & - & - & 72.25 & 72.24 \\
\hline Glycerol-2 & - & - & - & 69.72 & 69.80 \\
\hline Glycerol-3 & - & - & - & 66.72 & 66.72 \\
\hline $\mathrm{Glc} / \mathrm{Gal}^{* *}-1^{\prime}$ & - & - & 93.71 & 105.47 & 105.45 \\
\hline Glc/Gal-2' & - & - & 73.89 & 72.67 & 72.65 \\
\hline Glc/Gal-3' & - & - & 75.52 & 74.82 & 74.80 \\
\hline Glc/Gal-4' & - & - & 72.46 & 70.27 & 70.25 \\
\hline Glc/Gal-5' & - & - & 75.08 & 74.72 & 74.70 \\
\hline Glc/Gal-6' & - & - & 67.08 & 67.93 & 67.91 \\
\hline $\mathrm{Frc} / \mathrm{Gal}^{* * *}-1 "$ & - & - & 63.09 & 100.69 & 100.67 \\
\hline Frc/Gal-2" & - & - & 106.35 & 70.38 & 70.37 \\
\hline Frc/Gal-3" & - & - & 79.79 & 71.61 & 71.60 \\
\hline Frc/Gal-4" & - & - & 77.09 & 71.21 & 71.19 \\
\hline Frc/Gal-5" & - & - & 81.25 & 72.72 & 72.70 \\
\hline Frc/Gal-6" & - & - & 64.82 & 62.89 & 62.88 \\
\hline
\end{tabular}

*1, methyl linoleate; 2, linoleic aicd; 3, 6-linoleoyl- $\alpha$-D-Glucopyranosyl $\beta$-D-fructofuranoside; 4, 1-linolenoyl-3-( $\alpha$-D-galactopyranosyl (1 $\rightarrow 6)-\beta$-Dgalactopyranosyl) glycerol; 5, 1-linoleoyl-3-( $\alpha$-D-galactopyranosyl $(1 \rightarrow 6)-\beta$-D-galactopyranosyl) glycerol. **Glc, $\alpha$-D-glucopyranosyl (in compound 3); Gal, $\beta$-D-galactopyranosyl (in compounds 4 and 5); ***Frc, $\beta$-D-fructofuranosyl (in compound 3); Gal, $\alpha$-D-galactopyranosyl (in compounds 4 and 5). ${ }^{\#, \#}$ Exchangeable in same column

결합한 disaccharide로 확인되었다. 각 탄소 signal의 cheimcal shift로부터 $\alpha$-D-glucopyranose와 $\beta$-D-fructofuranose의 anomer 수산기가 서로 결합한 sucrose로 동정되었다. 비당부에서 유래 한 NMR signal을 보면 한개의 ester 탄소 $\left(\delta_{\mathrm{C}} 173.98, \mathrm{C}-1\right) 4$ 개의 olefin methine $\left(\delta_{\mathrm{C}} 130.78, \mathrm{C}-9 ; 128.75, \mathrm{C}-10 ; 128.75\right.$, C-12; 130.80, C-13; $\delta_{\mathrm{H}} 5.57, \mathrm{H}-9$; 5.48, H-10; 5.48, H-12; $5.57, \mathrm{H}-13), 12$ 개의 methylene $\left(\delta_{\mathrm{C}} 23.15-34.63\right.$; C-2 8, C-11, $\left.\mathrm{C}-14 \sim 17 ; \quad \delta_{\mathrm{H}} 1.22-2.91, \mathrm{H}-2 \sim 8, \mathrm{H}-11, \mathrm{H}-14 \sim 17\right)$, 1개의 methyl
$\left(\delta_{\mathrm{C}} 14.57, \mathrm{C}-18 ; \delta_{\mathrm{H}} 0.86, \mathrm{t}, J=6.6 \mathrm{~Hz}\right)$ 이 관측되어 octadecadienoic acid 로 추정되었다. NMR data를 문헌값[15]과 비교하 여 linoleic acid로 동정되었다. 지방산이 결합한 위치는 ${ }^{1} \mathrm{H}$ $\mathrm{NMR}$ 스펙트럼에서 $\alpha$-D-glucopyranose의 6 번 methylene 수소 signal의 chemical shift가 보통 $\delta_{\mathrm{H}} 4.0$ 인데 비하여 esterification 효과에 의해 저자장으로 이동하여 $\delta_{\mathrm{H}} 5.00,5.06$ 에서 관측된 점 으로부터 $\alpha$-D-glucopyranose의 6번 수산기와 linoleic acid의 carboxyl 기가 탈수축합한 것으로 판명되었다[15]. 이 사실은 
Table $2{ }^{1} \mathrm{H}$ NMR data of lipids from the rhizome of Cnidium officinalis Makino $\left(600 \mathrm{MHz}, \delta_{\mathrm{H}}\right.$, coupling pattern, $J$ in $\left.\mathrm{Hz}\right)$

\begin{tabular}{|c|c|c|c|c|c|}
\hline \multirow{2}{*}{ No of C } & \multicolumn{5}{|c|}{ Lipids* } \\
\hline & $1\left(\mathrm{CDCl}_{3}\right)$ & $\mathbf{2}\left(\mathrm{CDCl}_{3}\right)$ & $3\left(\mathrm{CD}_{3} \mathrm{OD}\right)$ & $4\left(\mathrm{CD}_{3} \mathrm{OD}\right)$ & $\mathbf{5}\left(\mathrm{CD}_{3} \mathrm{OD}\right)$ \\
\hline 2 & $2.26, t, 7.8$ & $2.32, \mathrm{t}, 7.5$ & $2.32, \mathrm{t}, 7.8$ & $2.36, \mathrm{t}, 7.5$ & $2.36, t, 7.5$ \\
\hline 3 & $1.58, \mathrm{tt}, 7.8,7.2$ & $1.61, \mathrm{tt}, 7.5,7.2$ & $1.58, \mathrm{tt}, 7.8,7.2$ & 1.62 , br.t, 7.5 & 1.62 , br.t, 7.5 \\
\hline 4 & 1.27 , overlapped & 1.29 , overlapped & 1.26 , overlapped & 1.34 , overlapped & 1.34 , overlapped \\
\hline 5 & 1.27 , overlapped & 1.29 , overlapped & 1.26 , overlapped & 1.34 , overlapped & 1.34 , overlapped \\
\hline 6 & 1.27 , overlapped & 1.29 , overlapped & 1.26 , overlapped & 1.37 , overlapped & 1.34 , overlapped \\
\hline 7 & 1.30, overlapped & 1.32 , overlapped & 1.34 , overlapped & 1.29 , overlapped & 1.37 , overlapped \\
\hline 8 & $2.01, \mathrm{dt}, 7.2,6.9$ & $2.03, \mathrm{dt}, 7.2,6.9$ & 2.09 , overlapped & $2.07, \mathrm{dt}, 9.6,6.0$ & $2.07, \mathrm{dt}, 9.6,6.0$ \\
\hline 9 & 5.38-5.32, overlapped & 5.38-5.34, overlapped & 5.57 , overlapped & 5.38 , overlapped & 5.36, overlapped \\
\hline 10 & 5.32-5.26, overlapped & 5.38-5.34, overlapped & 5.48 , overlapped & 5.38, overlapped & 5.32, overlapped \\
\hline 11 & $2.72, \mathrm{dd}, 6.9,6.9$ & $2.75, \mathrm{dd}, 6.6,6.6$ & 2.91 , overlapped & 2.81, br. dd, 5.7, 5.7 & 2.78, br. dd, $6.9,6.9$ \\
\hline 12 & 5.32-5.26, overlapped & 5.38-5.34, overlapped & 5.48 , overlapped & 5.34, overlapped & 5.32, overlapped \\
\hline 13 & 5.38-5.32, overlapped & 5.38-5.34, overlapped & 5.57 , overlapped & 5.34 , overlapped & 5.36 , overlapped \\
\hline 14 & $2.01, \mathrm{dt}, 7.2,6.9$ & $2.03, \mathrm{dt}, 7.2,6.9$ & 2.09 , overlapped & 2.81, br. dd, $5.7,5.7$ & $2.07, \mathrm{dt}, 9.6,6.0$ \\
\hline 15 & 1.30 , overlapped & 1.32 , overlapped & 1.34, overlapped & 5.31, overlapped & 1.37 , overlapped \\
\hline 16 & 1.23 , overlapped & 1.26 , overlapped & 1.22 , overlapped & 5.31 , overlapped & 1.30 , overlapped \\
\hline 17 & 1.23 , overlapped & 1.26 , overlapped & 1.22 , overlapped & 2.09, br. q, 7.8 & 1.30 , overlapped \\
\hline 18 & $0.85, \mathrm{t}, 6.6$ & $0.87, \mathrm{t}, 6.9$ & $0.86, \mathrm{t}, 6.6$ & $0.91, \mathrm{t}, 7.8$ & $0.91, \mathrm{t}, 6.9$ \\
\hline $\mathrm{OMe}$ & $3.62, \mathrm{~s}$ & - & - & - & \\
\hline Glycerol-1 & & & & $\begin{array}{l}3.85 \text {, overlapped } \\
3.65 \text {, overlapped }\end{array}$ & $\begin{array}{l}\text { 3.85, overlapped } \\
3.66 \text {, overlapped }\end{array}$ \\
\hline Glycerol-2 & & & & 3.98 , br. dt, $10.8,5.4$ & 3.99 , br. dt, $10.8,5.4$ \\
\hline Glycerol-3 & & & & $4.15, \mathrm{~d}, 5.4$ & $4.15, \mathrm{~d}, 5.4$ \\
\hline $\mathrm{Glc} / \mathrm{Gal}^{* *}-1^{\prime}$ & & & 6.21 , br.s & $4.25, \mathrm{~d}, 7.8$ & $4.25, \mathrm{~d}, 7.2$ \\
\hline Glc/Gal-2' & & & 4.20 , overlapped & $3.54, \mathrm{dd}, 7.8,9.6$ & $3.54, \mathrm{dd}, 7.2,9.6$ \\
\hline Glc/Gal-3' & & & $4.70, \mathrm{dd}, 9.0,9.0$ & $3.49, \mathrm{dd}, 9.6,3.0$ & $3.50, \mathrm{dd}, 9.6,3.0$ \\
\hline Glc/Gal-4' & & & 4.21 , overlapped & 3.87 , overlapped & 3.87 , overlapped \\
\hline Glc/Gal-5' & & & $4.80-4.79, \mathrm{~m}$ & 3.76 , overlapped & 3.76 , overlapped \\
\hline Glc/Gal-6' & & & $\begin{array}{l}5.06, \text { overlapped } \\
5.00, \text { overlapped }\end{array}$ & $\begin{array}{l}3.90 \text {, overlapped } \\
3.68 \text {, overlapped }\end{array}$ & $\begin{array}{l}\text { 3.91, overlapped } \\
3.68 \text {,overlapped }\end{array}$ \\
\hline $\mathrm{Frc} / \mathrm{Gal}^{* * *}-1 "$ & & & $\begin{array}{l}4.53, \mathrm{~d}, 12.0 \\
4.38, \mathrm{~d}, 12.0\end{array}$ & $4.86, \mathrm{~d}, 3.6$ & $4.87, \mathrm{~d}, 3.6$ \\
\hline Frc/Gal-2" & & & - & $3.79, \mathrm{dd}, 3.6,9.6$ & $3.79, \mathrm{dd}, 3.6,9.6$ \\
\hline Frc/Gal-3" & & & 5.01 , overlapped & 3.73 , overlapped & 3.74 , overlapped \\
\hline Frc/Gal-4" & & & 4.98 , overlapped & 3.89 , overlapped & 3.90 , overlapped \\
\hline Frc/Gal-5" & & & $4.65, \mathrm{~m}$ & 3.86 , overlapped & 3.86 , overlapped \\
\hline Frc/Gal-6" & & & $4.36, \mathrm{~d}, 10.2$ & 3.71, overlapped & 3.71, overlapped \\
\hline
\end{tabular}

*1, methyl linoleate; 2, linoleic aicd; 3, 6-linoleoyl- $\alpha$-D-Glucopyranosyl $\beta$-D-fructofuranoside; 4, 1-linolenoyl-3-( $\alpha$-D-galactopyranosyl (1 $\rightarrow 6)-\beta$-Dgalactopyranosyl) glycerol; 5, 1-linoleoyl-3-( $\alpha$-D-galactopyranosyl $(1 \rightarrow 6)-\beta$-D-galactopyranosyl) glycerol. **Glc, $\alpha$-D-glucopyranosyl (in compound 3); Gal, $\beta$-D-galactopyranosyl (in compounds 4 and 5); ***Frc, $\beta$-D-fructofuranosyl (in compound 3); Gal, $\alpha$-D-galactopyranosyl (in compounds 4 and 5)

$\mathrm{HMBC}$ 스펙트럼에서 ester 탄소 signal ( $\delta_{\mathrm{C}}$ 173.98)과 $\alpha$-Dglucopyranose의 6 번 methylene 수소 signal 사이에서 cross peak가 관측된 점으로부터 최종 확인되었다. 따라서 화합물 3은 6-linoleoyl- $\alpha$-D-glucopyranosyl $\beta$-D-fructofuranoside로 구조동정 되었다.

화합물 4는 positive FAB-MS 스펙트럼에서 분자이온피크가 $\mathrm{m} / \mathrm{z}$ 677에서 관측되어 분자량을 $676 \mathrm{Da}$ 로 결정하였다. IR 스
펙트럼에서 수산기 $\left(3343 \mathrm{~cm}^{-1}\right)$, ester $\left(1732 \mathrm{~cm}^{-1}\right)$ 및 olefin (1456, $1376 \mathrm{~cm}^{-1}$ ) 에서 유래한 흡수밴드가 확인되었다. NMR 스펙트 럼을 보면 모두 33 개의 탄소 signal 이 관측되었고, 각 signal 의 chemical shift로부터 2 분자의 hexose와 18 개로 이루어진 1 분자의 지방산과 1 분자의 glycerol로 이루어진 diglycosyl monoglyceride로 확인되었다. ${ }^{13} \mathrm{C}-\mathrm{NMR}$ 스펙트럼에서 당부에서 유래한 signal의 chemical shift로부터 말단 $\alpha$-D-galactopyranosyl 
의 존재가 확인되었다 $\left(\delta_{\mathrm{C}} 100.69, \mathrm{C}-1^{\prime \prime} ; 70.38, \mathrm{C}-2^{\prime \prime} ; 71.61\right.$, C-3", 71.21, C-4", 72.72, C-5", 62.89, C-6"). 또 다른 당에서 유래한 탄소 signal의 chemical shift를 보면, 1 개의 hemiacetal $\left(\delta_{\mathrm{C}} 105.47, \mathrm{C}-1^{\prime}\right), 4$ 개의 oxygenated-methine $\left(\delta_{\mathrm{C}} 72.67, \mathrm{C}-2^{\prime}\right.$; 74.82, C-3'; 70.27, C-4'; 74.72, C-5')은 $\beta$-D-galactopyranose 의 것과 같았으나, 1 개의 oxygenated-methylene 탄소 signal의 chemical shift는 보통의 값인 $\delta_{\mathrm{C}} 62$ 보다 저자장으로 이동되어 $\delta_{\mathrm{C}}$ 67.93으로 나타났다. 이는 $\beta$-D-galactopyranose의 6 번 수산기 에 다른 당이 결합하여 glycosidation 효과에 의해 저자장으로 이동된 것으로 확인되었다. 따라서 당부의 부분 구조는 $\alpha$-Dgalactopyranosyl $(1 \rightarrow 6)-\beta$-D-galactopyranose로 동정되었다. 지방 산의 경우 NMR signal을 보면 한개의 ester 탄소 $\left(\delta_{\mathrm{C}} 175.63\right.$, $\mathrm{C}-1)$ 6개의 olefin methine $\left(\delta_{\mathrm{C}} 132.88, \mathrm{C}-9\right.$; 128.40, C-10; 129.36, C-12; 129.35, C-13; 129.01, C-15; 131.24, C-16), 10 개의 methylene $\left(\delta_{\mathrm{C}} 21.64-35.07, \mathrm{C}-2 \sim 8, \mathrm{C}-11, \mathrm{C}-14, \mathrm{C}-\right.$ $17)$, 1 개의 methyl $\left(\delta_{\mathrm{C}} 14.81, \mathrm{C}-18\right)$ 이 관측되어 octadecatrienoic acid로 추정되었다. NMR data를 문헌값[15]과 비교하여 linolenic acid로 동정되었다. Linolenic acid의 결합위치는 ${ }^{1} \mathrm{H}-$ NMR 스펙트럼에서 glycerol의 3번 oxygenated-methylene 수소 signal이 보통보다 저자장으로 이동된 $\delta_{\mathrm{H}} 4.15$ 에서 관측된 점과, $\mathrm{HMBC}$ 스펙트럼에서 linolenic acid의 ester 탄소 signal $\left(\delta_{\mathrm{C}}\right.$ 175.63, C-1)이 glycerol의 3번 oxygenated-methylene 수소 signal과 correlation을 보인 점으로부터 glycerol의 3번 수산기로 확인되었다. 당의 결합위치는 $\mathrm{HMBC}$ 스펙트럼에서 $\beta$-Dgalactopyranose의 anomer 탄소 및 수소 signal $\left(\delta_{\mathrm{C}} 105.47, \delta_{\mathrm{H}}\right.$ 4.25)과 glycerol의 1 번 수소 및 탄소 signal $\left(\delta_{\mathrm{H}} 3.85,3.65\right.$, $\delta_{\mathrm{C}}$ 72.25)과의 사이에 cross peak가 관측된 점으로부터 glycerol 의 1 번 수산기로 확인되었다. 따라서 화합물 4는 1-linolenoyl3-( $\alpha$-D-galactopyranosyl $(1 \rightarrow 6)-\beta$-D-galactopyranosyl) glycerol로 구조동정되었다.

화합물 5는 positive FAB-MS 스펙트럼에서 분자이온피크가 $\mathrm{m} / z$ 679에서 관측되어 분자량을 $678 \mathrm{Da}$ 로 결정하였다. IR 스펙 트럼에서 수산기 $\left(3347 \mathrm{~cm}^{-1}\right)$, ester $\left(1732 \mathrm{~cm}^{-1}\right)$ 및 olefin (1648, $1377 \mathrm{~cm}^{-1}$ )에서 유래한 흡수밴드가 확인되었다. 화합물 5의 분 자량이 화합물 4 보다 $2 \mathrm{Da}$ 큰 점과, $\mathrm{NMR}$ 스펙트럼에서 지방 산을 제외한 signal이 화합물 $\mathbf{4}$ 와 유사한 점으로부터 화합물 $\mathbf{5}$ 는 지방산 구조에서 화합물 4 보다 이중결합이 한 개 적은 화합 물로 추정되었다. 지방산의 경우 NMR signal을 보면 1 개의 ester 탄소 $\left(\delta_{\mathrm{C}} 175.61, \mathrm{C}-1\right)$ 4개의 olefin methine $\left(\delta_{\mathrm{C}} 131.02\right.$, C-9; 129.25, C-10; 129.19, C-12; 131.08, C-13; $\delta_{\mathrm{H}} 5.36, \mathrm{H}-$ 9; 5.32, H-10; 5.32, H-12; 5.36, H-13), 12 개의 methylene $\left(\delta_{\mathrm{C}} 23.77-35.07, \mathrm{C}-2 \sim 8, \mathrm{C}-11, \mathrm{C}-14 \sim 17 ; \delta_{\mathrm{H}} 1.30-2.78, \mathrm{H}-\right.$ $2 \sim 8, \mathrm{H}-11, \mathrm{H}-14 \sim 17), 1$ 개의 methyl (14.59, C-18; $\delta_{\mathrm{H}} 0.91$, $\mathrm{H}-18$ ) 관측되어 octadecadienoic acid로 추정되었다. NMR data 를 문헌값[15]과 비교하여 linoleic acid로 동정되었다. 따라서 화합물 5는 1-linoleoyl-3-( $\alpha$-D-galactopyranosyl $\quad(1 \rightarrow 6)-\beta$-Dgalactopyranosyl) glycerol로 구조동정되었다.

Sucrose와 지방산이 결합한 화합물 3과 digalactosyl-monoglyceride 구조를 갖는 화합물 $\mathbf{4}$ 및 5는 천연에서 매우 드물게 발견되는 화합물이다. 또한, 보고된 지질화합물들의 면역증강, 항암활성 등을 통해 $[15,16]$, 천궁 근경의 다양한 약리활성 소재 로서의 가능성을 기대할 수 있다.
초 록

천궁의 근경을 $\mathrm{EeOH}$ 수용액으로 추출하였으며, 얻어진 추출물 을 용매계통분획방법을 이용하여 $\mathrm{EtOAc}$ 분획, $n-\mathrm{BuOH}$ 분획 및 물 분획으로 나누었다. EtOAc 분획과 $n-\mathrm{BuOH}$ 분획에 대하 여 silica gel, octadecyl silica gel 및 Sephadex LH-20을 정지 상으로 사용하여 칼럼크로마토그래피를 반복 수행한 결과 5종 의 lipid를 분리하였다. 5 종 화합물은 IR, MS 및 $\mathrm{NMR}$ 데이터 를 해석하여 각각 methyl linoleate (1), linoleic aicd (2) 6linoleoyl- $\alpha$-D-glucopyranosyl $\beta$-D-fructofuranoside (3), 1-linolenoyl3-( $\alpha$-D-galactopyranosyl $(1 \rightarrow 6)-\beta$-D-galactopyranosyl) glycerol (4) 및 1-linoleoyl-3-( $\alpha$-D-galactopyranosyl $(1 \rightarrow 6)-\beta$-D-galactopyranosyl) glycerol (5)로 구조동정하였다, 화합물 1과 3-5는 천궁에서는 이 번 연구에서 처음 분리되었다. 또한 화합물 1 과 2 의 경우 $\mathrm{NMR}$ 데이터가 완전하지 않거나, 문헌에 따라 서로 다르게 보고되어 있으나, 이번에 2-D NMR 등을 자세히 검토하여 모든 탄소 및 수소에 대한 데이터를 확실하게 동정하였다. Sucrose와 지방산 복합체인 화합물 3과 digalactosyl monoglyceride인 화합물 $\mathbf{4}$ 와 $\mathbf{5}$ 는 현재까지 천연에서 매우 드물게 보고되어 있다. 보고된 지질화합물들의 면역증강, 항암활성 등을 통해, 천궁 근경의 다 양한 약리활성 소재로서의 가능성을 기대할 수 있다.

Keywords 지질 · 천궁 · Diglycosyl monoglyceride · Linoleoyl sucrose $\cdot$ Nuclear magnetic resonance

감사의 글 본 성과물은 농촌진흥청 연구사업(세부과제번호: PJ01420403) 의 지원에 의해 이루어진 것입니다. 지원에 감사드립니다.

\section{References}

1. Li W, Tang Y, Chen Y, Duan JA (2012) Advances in the chemical analysis and biological activities of Chuanxiong. Molecules 17: 1061410651. doi: 10.3390/molecules 170910614

2. Zhou J, Qu F (2009) Treating gynaecological disorders with traditional chinese medicine: A Review. Afr J Tradit Complement Altern Med 6: 494-517. doi: 10.4314/ajtcam.v6i4.57181

3. Shin HY, Lee SH, Kim HR, Kim JH, Yang SB, Cho SY, Park JM, Ko CN, Park SU (2018) A review of clinical research trends in the treatment of primary headache disorders with pharmacopuncture. J Int Korean Med 39: 1191-1205. doi: 10.22246/jikm.2018.39.6.1191

4. Lee KY, Kim JH, Kim EY, Yeom M, Jung HS, Sohn Y (2019) Water extract of Cnidii Rhizoma suppresses RANKL-induced osteoclastogenesis in RAW 264.7 cell by inhibiting NFATc1/c-Fos signaling and prevents ovariectomized bone loss in SD-rat. BMC Complement Altern Med 19: 207-219. doi: 10.1186/s12906-019-2611-8

5. Cho SK, Kwon OI, Kim CJ (1996) Anti-inflammatory and analgesic activities of the extracts and fractions of Cnidii Rhizoma. Kor J Pharmacogn 27: 282-287

6. Sim Y, Shin S (2014) Antibacterial activities of the essential oil from the leaves and rhizomes of Cnidium officinale Makino. J Essent Oil Res 26: 452-457. doi: 10.1080/10412905.2014.951456

7. Jeong JE, Lee YJ, Choi YA, Park JM, Lee SM, Jo NY, Lee EY, Lee CK, Roh JD (2021) Seizure after subdural hematoma treated with combination Western-Korean medicine. J Acupunct Res 38: 72-78

8. Jeong JB, Ju SY, Park JH, Lee JR, Yun KW, Kwon ST, Lim JH, Chung GY, Jeong HJ (2009) Antioxidant activity in essential oils of Cnidium officinale makino and Ligusticum chuanxiong hort and their inhibitory 
effects on DNA damage and apoptosis induced by ultraviolet B in mammalian cell. Cancer Epidemiol 33: 41-46. doi: 10.1016/j.canep. 2009.04.010

9. Lee JT, Park JH, Lee KH (2005) Effect of methanol extract of Cnidii rhizoma on the function of receptors for GABA and glycine. J Korean Acad Pediatr Dent 32: 55-66

10. Kim SD, Kim GW, Shin HM (2006) Effects of Cnidium officinale, Petasites japonicas, Coptis chinonsis extract mixture on vasodilation. Korean J Orient Physiol \& Pathol 20:1620-1624

11. Lee SY, Kim MJ, Yim DS, Chi HJ, Kim HS (1990) Phthalide content of Cnidium rhizome. Saengyak Hakhoechi 21: 69-73

12. Cao YM, Guo DL, Jin MY, Tan L, Yang TL, Deng F, Gu YC, Li XH, Cao ZX, Deng Y (2020) Two new nor-sesquiterpenoids from Fusarium tricinctum, an endophytic fungus isolated from Ligusticum chuanxiong. Nat Prod Res: Ahead of print. doi: 10.1080/14786419.2020.1712385

13. Xiao W, Ni F, Wen J, Xie X, Li M, Wu Y, Wang Z (2020) Extraction and purification of (E)-3-(4-hydroxy-3-methoxyphenyl)glyceryl ferulate from Ligusticum chuanxiong as anti-inflammatory or anti-complement agent. China patent: CN110857271

14. Lee TF, Lin YL, Huang YT (2007) Studies on antiproliferative effects of phthalides from Ligusticum chuanxiong in hepatic stellate cells. Planta Med 73: 527-534. doi: 10.1055/s-2007-981520

15. Ko JH, Castaneda R, Joo SW, Kim HG, Lee YG, Lee YH, Kang TH, Baek NI (2018) Glycerides isolated from the aerial parts of Malva verticillata cause immunomodulation effects via splenocyte function and NK antitumor activity. Food Sci Biotechnol 27: 1023-1030. doi: 10.1007/s10068-018-0344-y

16. Ko JH, Cho SM, Joo SW, Kim HG, Lee YG, Kang SC, Baek NI (2018) Glycosyl glycerides from the aerial parts of Malva verticillata and their chemopreventive effects. Bioorg Chem 78: 381-392. doi: 10.1016/ j.bioorg.2018.03.013 